\title{
T2K Near Detector Tracker
}

\author{
Flor de María Blaszczyk ${ }^{* \dagger}$ \\ CEA, Centre de Saclay / Irfu / SPP \\ E-mail: flordemaria.blaszczykecea.fr
}

Tokai to Kamioka (T2K) is a new generation neutrino oscillation experiment in Japan that started collecting data in 2009. A near detector (ND280) placed in a $0.2 \mathrm{~T}$ magnetic field is located at $280 \mathrm{~m}$ from the target to allow the characterisation of the neutrino beam before oscillation. In particular, the detector measures neutrino energy spectra, beam flavor composition, backgrounds and cross-sections. An essential element in ND280 is the tracker, which is composed of two fine grained detectors (FGD) that serve as targets for neutrino interactions and measure cross-sections, and three time projection chambers (TPC) that track and identify charged particles. The TPCs' readout planes are instrumented with Micromegas micro-pattern detectors. The first FGD is made of scintillator bars only whereas the second one includes water targets. The performance of the tracker with real data is presented.

35th International Conference of High Energy Physics

July 22-28, 2010

Paris, France

* Speaker.

${ }^{\dagger}$ on behalf of the $\mathrm{T} 2 \mathrm{~K}$ Collaboration 


\section{The T2K Experiment}

Tokai to Kamioka (T2K) is a new generation long baseline neutrino oscillation experiment in Japan. T2K will measure $\theta_{13}$, one of the last unknown parameters of the Pontecorvo-MakiNakagawa-Sakata matrix, or at least improve the existing upper limit on $\sin ^{2} 2 \theta_{13}$ by an order of magnitude with the appearance of $v_{e}$ in the $v_{\mu}$ beam. Using $v_{\mu}$ disappearance, T2K will also measure precisely the atmospheric oscillation parameters $\Delta \mathrm{m}_{23}^{2}$ and $\theta_{23}$.

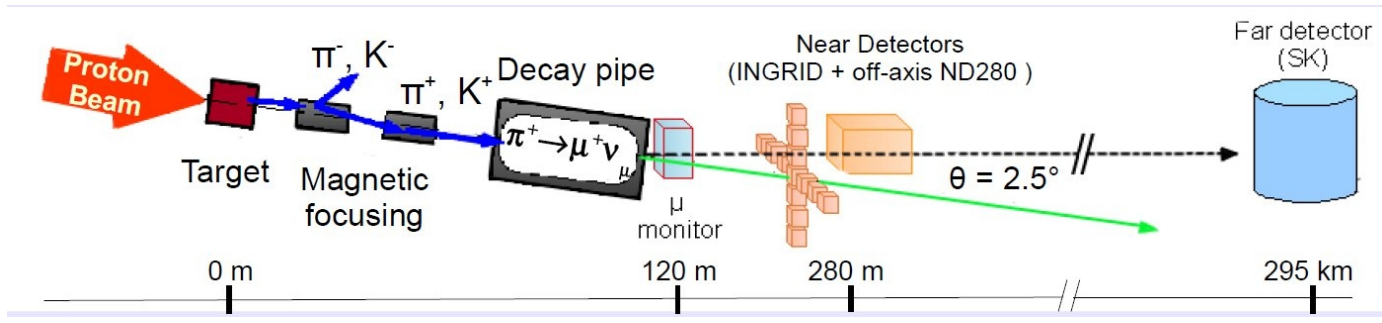

Figure 1: Tokai to Kamioka baseline.

The $v_{\mu}$ beam is produced at J-PARC (Tokai) by a $30 \mathrm{GeV}$ proton beam colliding onto a carbon target (Fig.1). Prior to colliding onto the target, the proton beam is monitored by the Optical Transition Radiation detector. After the decay pipe, a muon detector monitors the neutrino beam going towards the near detector ND280. This near detector, located at $280 \mathrm{~m}$ from the target, is made of one on-axis detector, INGRID, and one off-axis detector. The beam reaches the off-axis far detector Super Kamiokande, a 50 kT water Čerenkov detector at Kamioka, 295 km away.

The T2K first beam was commissioned in April 2009 and the experiment's first physics run started in January 2010.

\section{The off-axis near detector}
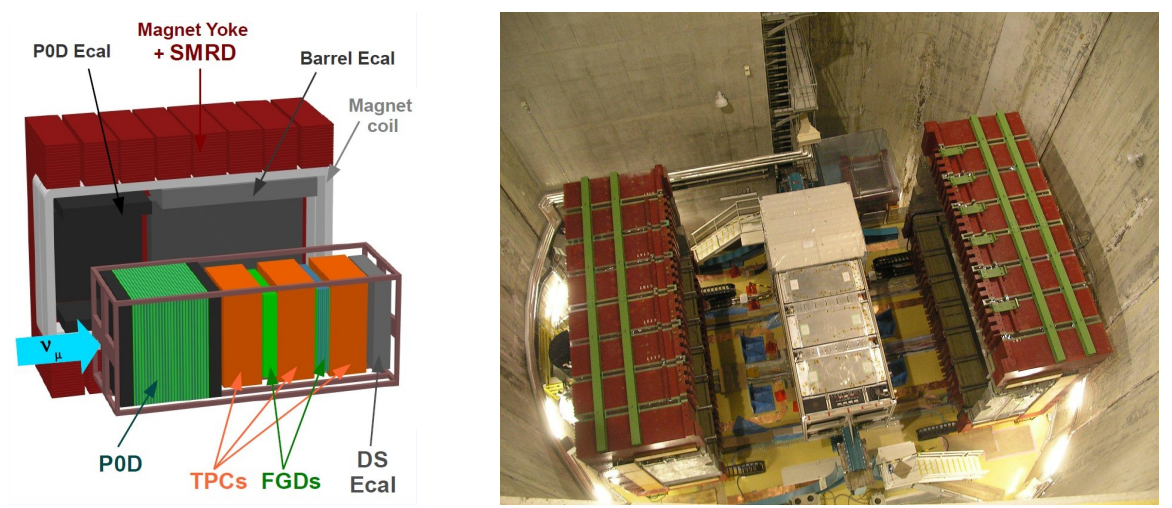

Figure 2: The off-axis near detector structure at J-PARC, Tokai.

Located at $280 \mathrm{~m}$ from the target with an off-axis angle of 2.5 degrees, the off-axis near detector characterizes the neutrino beam before oscillation. This means measuring various quantities 
such as beam flux, composition, direction, neutrino energy spectra, and cross sections. The detector is placed within a magnet refurbished from the UA1 experiment which provides a $0.2 \mathrm{~T}$ magnetic field.

The off-axis ND280 is composed by a subset of different detector types: a $\pi^{0}$ detector (P0D) and a tracker, surrounded by an electromagnetic calorimeter and side muon range detectors (SMRD) embedded within the magnet yoke (Fig.2). All parts but the barrel electromagnetic calorimeter were successfully installed, commissioned at the end of 2009, and have taken data for the first physics run. The magnet is operational and has been field mapped. The barrel electromagnetic calorimeter will be installed during summer 2010 .

\section{The tracker}

The off-axis ND280 tracking system is composed of three Time Projection Chambers (TPCs) and two Fine Grained Detectors (FGDs). Its main goals are the study of charged current interactions and the measurement of the beam $v_{e}$ contamination. These detectors have taken neutrino beam data as well as cosmic data for calibration purposes.

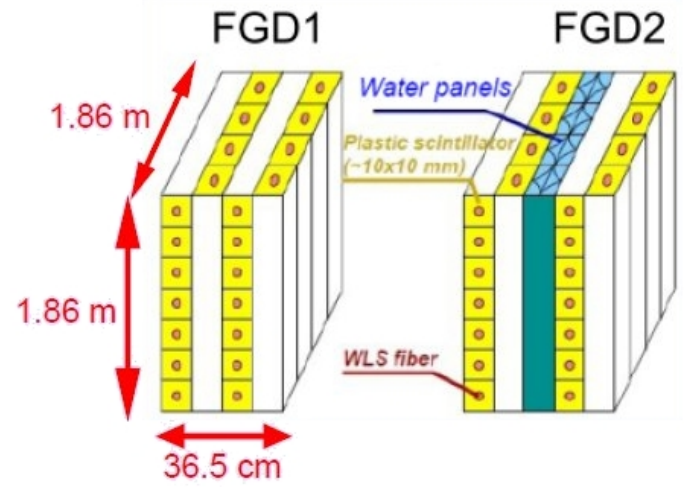

(a)

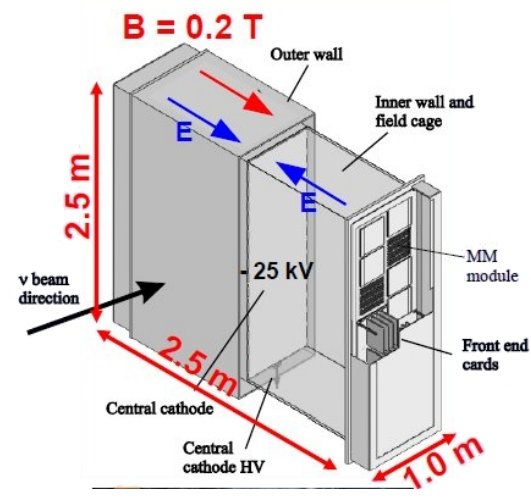

(b)

Figure 3: (a) Schematic FGD design and (b) TPC design.

\subsection{Fined Grained Detectors}

The fine grained detectors main purposes are to provide target mass for neutrino interactions and to measure neutrino cross sections on water and carbon. They are a crucial element for vertex reconstruction and will also allow tracking as well as particle identification.

Design - Both FGDs are made of thin scintillator bars $\left(9.61 \times 9.61 \times 1864 \mathrm{~mm}^{3}\right)$ arranged in horizontal and vertical layers to allow tracking (30 layers for the first FGD and 14 for the second one, Fig. 3(a)). Each bar yields an average of 30 photo-electrons per minimum ionizing particle. The second FGD has additional passive water targets to measure neutrino cross-section on water. The scintillation light produced in the bars is collected by a wavelength shifting fiber $(\varnothing=1 \mathrm{~mm})$. To 
improve light collection, one fiber tip has been mirrored while the other is connected to a multipixel photon counter (MPPC). Front-end electronics is based on the AFTER ASIC chip. A LED based light injection system is used for calibration purposes.

The MPPCs, specifically designed for T2K, are divided into 667 active pixels each $\left(50 \times 50 \mu \mathrm{m}^{2}\right.$ pixels) and have low dark noise [1]. It is the first time that these devices are used in such a large scale: all scintillator-based near detectors in T2K use this technology for fiber signal readout, which means over 52000 MPPCs including the barrel ECAL. These MPPCs have a photon detection efficiency of about $30 \%$ at nominal voltage.

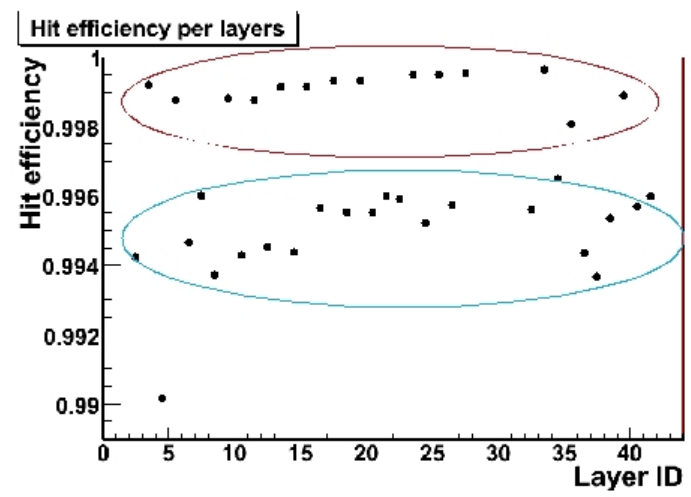

(a)

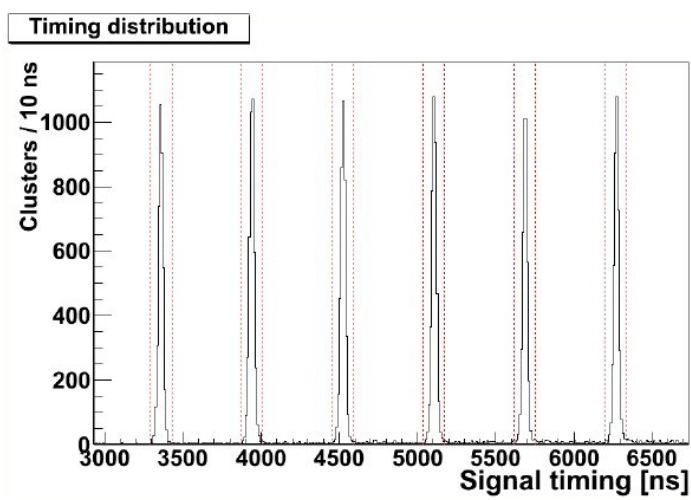

(b)

Figure 4: (a) Hit efficiency for horizontal (red) and vertical (blue) layers. (b) Timing resolution.

FGD performance - An important calibration result is the hit efficiency, measured with through going cosmics (Fig.4(a)). Hit efficiency is defined as the number of hits divided by the total number of layers crossed. The measurement shows an efficiency higher than $99 \%$ for all layers. The slightly higher efficiency for horizontal layers with respect to the vertical ones is due to a geometrical effect as cosmic rays are mainly downward going.

One of the first results obtained with neutrino data was the reconstructed timing of the events in the FGD (Fig.4(b)). The neutrino beam delivered by J-PARC has six bunches per spill, with an inter-bunch time of $581 \mathrm{~ns}$. This structure can be clearly seen in the FGDs, with almost no background in-between bunches.

\subsection{Time Projection Chambers}

The time projection chambers purposes are the 3D reconstruction of charged particles tracks, particle identification, and momentum measurement through track curvature induced by the magnetic field. The deposited energy $(\mathrm{dE} / \mathrm{dx})$ measurement used for particle identification is important to distinguish muons from electrons and protons from pions, hence its resolution must be better than $10 \%$. The physics requirements on momentum measurement are a resolution of $10 \%$ at $1 \mathrm{GeV}$ and an absolute momentum scale known at the $2 \%$ level.

Design - TPCs have a double-wall structure to allow better isolation and field homogeneity. They are filled with a $95 \% \mathrm{Ar} / 3 \% \mathrm{CF}_{4} / 2 \% \mathrm{iC}_{4} \mathrm{H}_{10}$ gaz mixture. Each TPC, divided in two drift 
regions by a central cathode, has two readout planes (Fig.3(b)). The latter are instrumented with bulk-Micromegas micro-pattern gaseous detectors [2]. There is a total of 72 Micromegas modules $\left(35 \times 36 \mathrm{~cm}^{2}\right.$ each) in all 3 TPCs (12 per readout plane) corresponding to a total active surface of about $9 \mathrm{~m}^{2}$. These are the largest TPCs ever instrumented with such devices. Each module is divided into 48 rows and 36 columns with a pad pitch of $7.0 \times 9.8 \mathrm{~mm}^{2}$. The average gain is about $10^{3}$ at a voltage of $-350 \mathrm{~V}$ and for a $128 \mu \mathrm{m}$ gap.

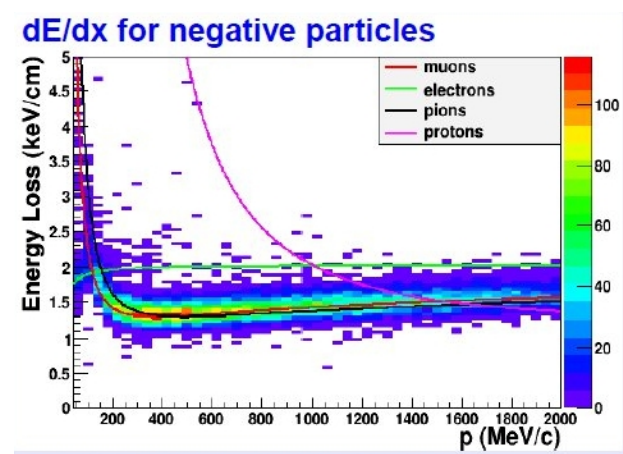

(a)

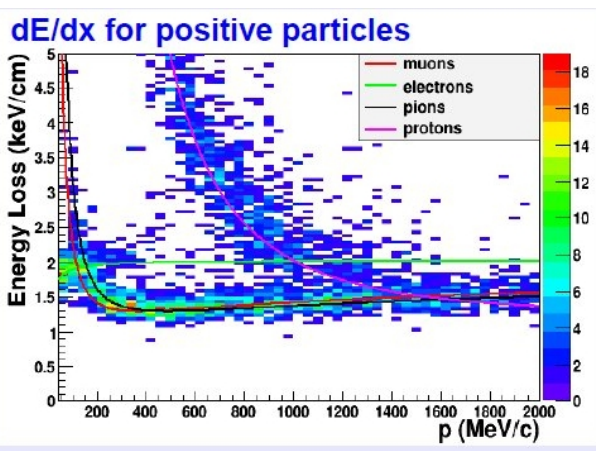

(b)

Figure 5: Deposited energy versus momentum for (a) negative particles and (b) positive particles.

TPC performance - All Micromegas modules and electronics were tested prior to their installation on a test bench at CERN where they proved to have a stable performance with an average response uniformity of $2 \%$. Also, each TPC was tested at TRIUMF (Canada) with beam $(\pi, \mathrm{e}, \mu)$. The test results show that the spatial resolution is $650 \mu \mathrm{m}$ at a $75 \mathrm{~cm}$ drift distance, which is good enough to obtain the required momentum resolution.

One of the first measurements done with the J-PARC neutrino data is the charged particles' deposited energy. $\mathrm{dE} / \mathrm{dx}$ is computed with a truncated mean method and its measured resolution is $7.8 \pm 0.2 \%$. The data sample studied contains mainly negatively charged muons (Fig.5(a)), positively charged pions and protons (Fig.5(b)).

\section{Conclusion}

$\mathrm{T} 2 \mathrm{~K}$ beamline and near detectors were successfully built and commissioned. The first physics run took place from January until June 2010 and data taking will resume at the end of the year. The near detectors are the first detectors using large numbers of MPPCs and having large-sized TPCs instrumented with Micromegas modules. These detectors have proved to work as expected and the performance required for the $\mathrm{T} 2 \mathrm{~K}$ physics program has been reached. More refined studies are under way, in particular physics related analyses.

\section{References}

[1] F. Retière et al., Nucl. Instrum. Meth. A610, 378-380 (2009);

[2] S. Anvar et al., Nucl.Intrum. Meth. A602, 415-520 (2009) 